\title{
ANXIETY AND AN AUTONOMIC REACTION TO PAIN
}

\author{
BY
}

\author{
MALCOLM PIERCY, ALICK ELITHORN, R. T. C. PRATT, and MARGARET CROSSKEY
}

From the Department of Psychological Medicine and the Neurological Research Unit of the Medical Research Council, National Hospital, Queen Square, London, and the Department of Experimental

Psychiatry, University of Birmingham

In a previous communication Elithorn, Piercy, and Crosskey (1955) reported changes in autonomic reactivity which followed prefrontal leucotomy. The change in reactivity which was observed was related to the kind of stimulus which elicited the reponse. It was suggested that the autonomic reactions in a painful situation were changed by leucotomy in that there was a marked reduction of the anticipatory component which appears in association with subjective anxiety.

Although the evidence suggested that most of the differences which were observed were directly related to the post-operative changes in the clinical picture, the surgical intervention might have modified autonomic activity in a way which was not closely related to behaviour or to subjective experience. In order further to elucidate the relations which obtain between patterns of autonomic reaction and emotional mechanisms we have undertaken a study of psychiatric patients using a "pain expectancy" technique similar to that used in the studies of leucotomized patients. The autonomic responses occurring with pain and with the anticipation of pain have a more obvious relationship with anxiety than with any other major psychiatric symptom. Accordingly, in this study the guiding principle in interpreting individual differences in autonomic reaction has been the extent to which anxiety was a clinically prominent symptom.

\section{Experimental Methods}

The apparatus and procedure employed in this investigation for measuring autonomic reaction was essentially the same as that described in the previous communication and is now described only in outline.

Changes in the electrical resistance of the palmar skin (psychogalvanic responses) were recorded graphically $(a)$ following an electrical stimulus to the little finger and $(b)$ following a red light which was exhibited for a short interval (about 400 millisec.) eight seconds before the onset of the electrical stimulus. Successive electrical stimuli were increased in intensity in regular steps from a subliminal value to the maximum intensity the subject would tolerate. Preliminary tests were carried out with each subject to determine the approximate intensities at which the stimulus was first perceived and at which it became as much as the subject said he could bear. The voltage increment for successive stimuli was then calculated for the individual subject so that approximately 16 supraliminal stimuli were administered before the subject said "stop". The procedure was explained to the subject before the experiment began and he was asked to say "stop" when the electrical stimulus became " too painful ". No further electrical stimuli were given after the subject said "stop", and this was made clear to the subject from the outset. After the subject had said "stop" the red light was exhibited three more times, but it was not followed by a shock. After he had said "stop" the subject was told to expect this. Measurements were also taken of the resting level of skin resistance, skin temperature, and environmental temperature. A graphic record of respiration was also kept and the responses to three maximal inspirations were measured before the start of the main investigation. For this the following procedure was adopted : the subject was told that a white light would flash at 3-second intervals and was asked to count the flashes subvocally and to take a deep breath on every tenth flash. This course was followed to ensure that the response was essentially to the stimulus provided by the respiratory excursion and not merely to the sound of the examiner's voice.

\section{Subjects}

Thirty-six psychiatric patients were examined with this technique, and the Table lists the diagnoses and ratings for the prominence of anxiety as a symptom, together with the resting levels of skin resistance and the mean responses to the three stimuli. The diagnoses and ratings for anxiety were made by one of the authors (R.T.C.P.) in the course of a clinical interview. Patients were rated for anxiety on a four-point scale. Ten patients 
TABLE

ANXIETY RATING, INITIAL SKIN RESISTANCE, AND MEAN LIGHT, SHOCK, AND DEEP BREATH RESPONSES

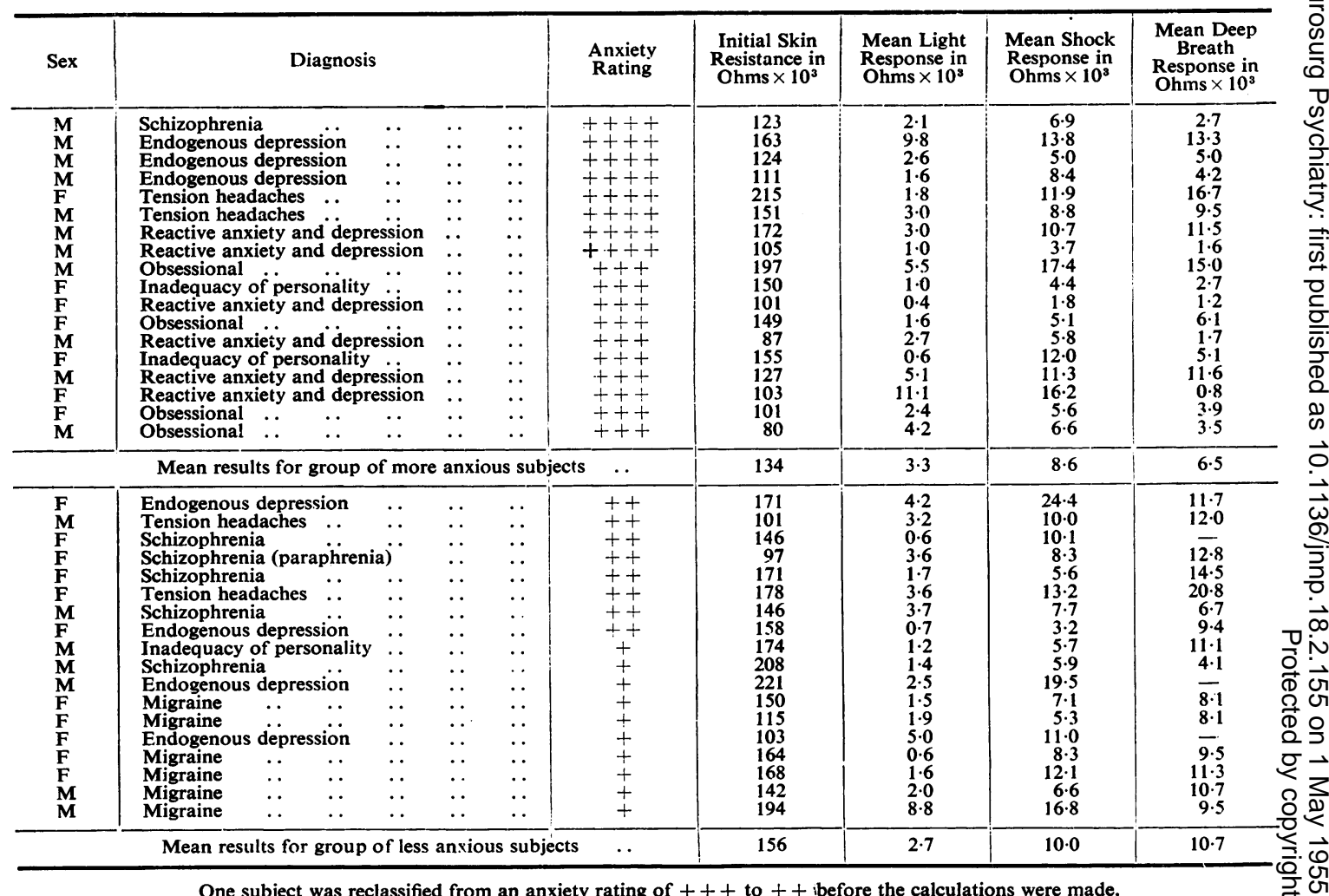

were rated + (least anxious), seven as,++ 11 as +++ , and eight as ++++ (most anxious). For purposes of analysis we wished to divide the 36 patients into two equal groups : less anxious and more anxious. It was therefore decided to reclassify one of the patients rated +++ to $a++$ rating. The author responsible for psychiatric assessment therefore reclassified the patient he considered to be the least anxious of the original +++ group. Patients were not examined as to their autonomic reactivity until after the diagnosis and rating for anxiety had been decided. Each subject was tested with the procedure described above on one occasion only.

\section{Experimental Design and Methods of Analysis}

In the study of the anticipation of pain following prefrontal leucotomy we calculated an " expectancy ratio" for each subject. This ratio expressed the quantitative relationship between the autonomic responses to the two stimuli, namely, an electrical stimulus and a red light which regularly preceded the electrical stimulus. This ratio was found to change following leucotomy in that the response to the red light became comparatively smaller. It was considered that this change was compatible with decreased anxiety or decreased anticipation of pain. In that study the expectancy ratio was obtained from the mean responses to the two classes of stimuli and the effect of the intensity of the electrical stimulus on the ratio or on the size of the individual responses was largely ignored. This was possible since the relative change in autonomic reactivity to the two kinds of stimulus following leucotomy was fairly gross and was readily identified in terms of the expectancy ratio.

In the present investigation we have used a modified form of this expectancy ratio and have also employed other numerical criteria which, on theoretical grounds, we believed might reflect individual differences in anxiety or anticipatory tension. As we pointed out previously, it is difficult to interpret psychologically differences between the absolute magnitude of responses in different subjects 
or in the same subject on different occasions. The magnitude of a sudomotor response will depend not only upon the experimental variable which is introduced but also upon the state of heat balance, and the latter cannot be controlled without extreme practical difficulties. In investigating these autonomic reactions we have therefore used numerical relationships (usually ratios) between responses to different stimuli, for example, electrical stimulus and warning light, or electrical stimuli of different intensities; or relationships between responses to a stimulus of varying significance, for example, response to a light preceding a weak electrical stimulus and response to the same light preceding an intense electrical stimulus. Since successive electrical stimuli increased in regular steps, the change in the significance of the warning light during the course of the experimental session may be assumed to be directly related to the intensity of the preceding electrical stimulus.

As described above, the patients assessed at the clinical interview and subsequently serving as subjects for this investigation were divided into two equal groups : more anxious and less anxious. It is the differences in the autonomic reaction of these two groups which are reported below. It should be mentioned that the differences in autonomic reaction which we sought to identify in this study were of a much finer degree than those which occurred in patients undergoing prefrontal leucotomy. In the latter the clinical change was usually from a severely disabling neurotic state to a state of greatly reduced anxiety, and in some cases to a state of abnormally low anxiety. In this study we are examining differences between two groups of psychiatric patients, one group being judged clinically to be more anxious than the other. But since both groups consisted of psychiatric patients, both were almost certainly more anxious than a normal population. We are thus distinguishing between degrees of pathological anxiety.

\section{Results}

In the Table are shown the skin resistance levels and the mean size of responses for each patient, together with the mean levels and responses for each group of subjects. The magnitude of the response to the electrical stimulus ("shock response") is taken as the greatest fall in skin resistance which occurs following the presentation of the paired light and shock stimuli, the level immediately before the presentation of the light being the base line for the response. The magnitude of the response to light ("light response") is taken as the greatest fall in skin resistance which occurs following the exhibition of the light and preceding the occurrence of the electrical stimulus. It can be seen that the mean resistance level of the more anxious group is lower (corresponding to a higher rate of sweating) than that of the less anxious group. The mean responses to shock and to a deep breath are greater in the less anxious group and the mean response to light is greater in the more anxious group. In addition to the initial resting level, the skin resistance was recorded throughout the session, the general tendency being for the levels to fall as the session proceeded. The mean level of skin resistance of the more anxious group fell $29 \%$ from $134 \times 10^{3}$ ohms to $95 \times 10^{3}$ ohms and the mean level of the less anxious group fell $36 \%$ from $156 \times 10^{3}$ ohms to $100 \times 10^{3}$ ohms. For the two groups these various measures of autonomic reactivity differ in sign as well as in extent and, with the exception of the responses to the deep breaths, none of the differences attains an acceptable level of statistical significance. Such differences between the two groups in the absolute size of the responses would in any case be difficult to interpret because the part played by the state of heat balance of the subjects is unknown.

\section{Expectancy Ratios}

The " expectancy ratio", being largely independent of heat-regulating requirements, provides a more meaningful measure. This is expressed here as the mean reponse to light divided by the mean response to shock. This ratio has been multiplied by 100 to give a percentage rating for " expectancy" and has been calculated for each of the 36 patients. The mean value of this measure of expectancy is higher in the more anxious (31.2) than in the less anxious group (23.4) but the difference does not attain statistical significance. This measure, which was adequate to identify the effect of leucotomy, is evidently too coarse to reveal the differences between our present two groups of psychiatric patients. Accordingly, we sought to employ a ratio which has as its denominator the response to a stimulus of minimal emotional significance. It would seem reasonable to suppose that for both the more anxious and the less anxious patients a maximal inspiration made in accordance with instructions previously given by the examiner would be of minimal emotional significance and that, in both groups, the fall in skin resistance which follows a deep breath would have a considerably smaller " psychological" component than the autonomic responses which follow either the electrical stimulus or the warning light. We have accordingly used the mean response to a deep 


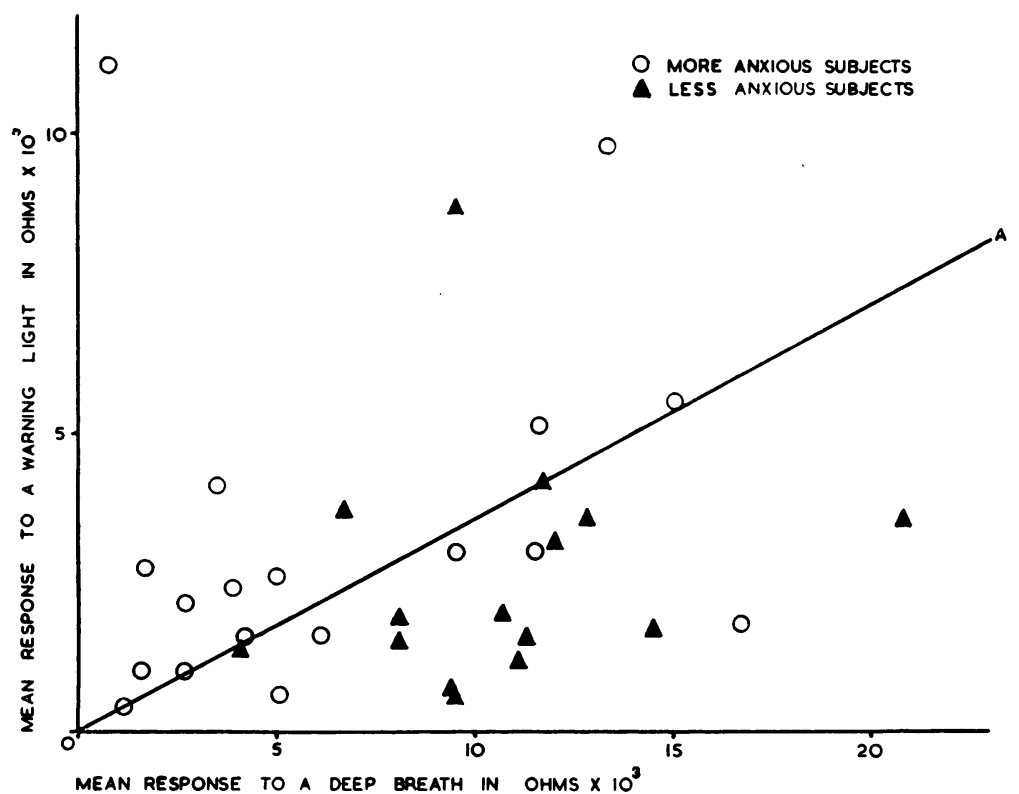

FIG. 1

breath in each subject as a denominator against which to measure the magnitude of the mean responses to warning light and shock. In so far as the response to one kind of stimulus is used as a yardstick against which to measure the response to another kind of stimulus, these two new ratios (response to light/response to deep breath ; response to shock/response to deep breath) are not essentially different from the expectancy ratio, the latter being useful in identifying the effect of leucotomy but not providing a significant difference between the two groups in the present study.

For the more anxious group the mean for the ratio shock response/deep breath response was 1.9 ; for the less anxious group the mean for the same ratio was $\mathbf{0 \cdot 8}$. The difference between the two groups is statistically significant at the 0.01 level of confidence $(t=3.33, N=33){ }^{*}$

The mean for the ratio light response/deep breath response was 0.5 in the more anxious group and 0.2 in the less anxious group. This difference between the two groups is significant at the 0.01 level of confidence $(t=2 \cdot 579, N=33)$. Thus, if the autonomic response to a maximal inspiration is regarded as a measure of "physiological" autonomic reactivity and is used as a base line or denominator, then the responses both to warning

\footnotetext{
* The mean ratios derived from a group of individual comparisons are the geometric means and the $t$ tests of significance between such means have been made after a logarithmic transformation of the original ratios. Because three subjects gave unsatisfactory deep inspirations their results have had to be omitted hence $\mathrm{N}=33$ instead of 36 .
}

light and to shock are relatively greater in the more anxious than in the less anxious group.

The light response/deep breath response ratios are shown graphically in Fig. 1 . The ordinate and the abscissa represent the magnitude of response to warning lights and to deep breaths respectively and the position of the circles and triangles shows the relationship between the mean response to the warning light and the mean response to a deep breath in the case of each subject. A line has been drawn to represent the mean ratio for the whole group and it can be seen that there is a tendency for the more anxious subjects to be clustered above the line showing comparatively greater responses to light, and for the less anxious subjects to be clustered below the line showing comparatively greater responses to a deep breath. This diagram shows that in this group of patients there was in $24(72 \cdot 7 \%)$ of the 33 subjects concordance between the autonomic and clinical criteria of anxiety.

\section{Pain Tolerance}

For each subject a record was kept of the lowest perceptible voltage at the finger tips and the voltage at which the subject said "stop" when the intensity of successive electrical stimuli was increased in small steps. The ratio of the highest voltage tolerated to the smallest perceived was designated the "pain tolerance ratio". The mean value for this tolerance ratio in the more anxious group was 4.82 (range 1.91 to 10.51 ) and in the less anxious group 4.83 (range 2.45 to 6.89). These two values do not differ significantly. It is therefore most unlikely that any of the autonomic criteria of anxiety reported above which are found to distinguish between the more anxious and the less anxious groups could be accounted for in terms of the intensity of the electrical stimulus tolerated by the two groups.

\section{Effect of Intensity of Electrical Stimulus on Response to Warning Light}

The position in serial order of any light stimulus corresponds to the intensity of the electrical stimulus which the light presages, so any habituation which 
occurs with repetition of the light stimulus is achieved in the face of the increasing emotional significance of the light stimulus. It is not surprising, therefore, that in both groups of patients the mean response to light at the end of the session is greater than the mean response to light at the beginning of the session. There is, however, a difference between the two groups. The responses of each subject have been divided into quarters corresponding to their serial order in the test session (see Fig. 2) and the measure we have used is the ratio of the mean response to light in the fourth quarter to the mean response to light in the first quarter. This has been calculated for each subject, and the mean value of this measure for the more anxious group is 2.4 and for the less anxious group $1 \cdot 3$. This difference between the two means is significant at the 0.05 level of confidence $(\mathrm{t}=2 \cdot 33, \mathrm{~N}=36)$. Thus the autonomic responses to the warning light of the less anxious group are less affected by increase in the intensity of the electrical stimulus which follows the light than are the corresponding responses of the more anxious group. A similar ratio was calculated for the responses to the electrical stimuli. For the more anxious group the mean of the ratios fourth quarter/first quarter was 3.8 and for the less anxious group $2 \cdot 8$. The two means are not significantly different but the effect is in the same direction as for the responses to the light.

In Fig. 2 the mean responses of each group to the two stimuli are shown for each of the four quarters. It can be seen that the tendency for responses to light to increase from the first to fourth quarter is more marked in the case of the more anxious group. In the case of responses to the electrical stimulus this difference is less marked and the responses of both groups increase from the first to fourth quarter. Fig. 3 shows the individual responses to light and to shock of two patients ; $3 \mathrm{a}$ is typical of the more anxious group and $3 \mathrm{~b}$ is typical of the less anxious group. In Fig. 3a the responses to light tend to increase with successive stimuli whereas in Fig. 3b some habituation is apparent. Also it can be seen that in Fig. 3a the response to light constitutes a greater proportion of the total response to shock than is the case in Fig. 3b. This effect corresponds to the difference in the "expectancy atios" of the two groups.

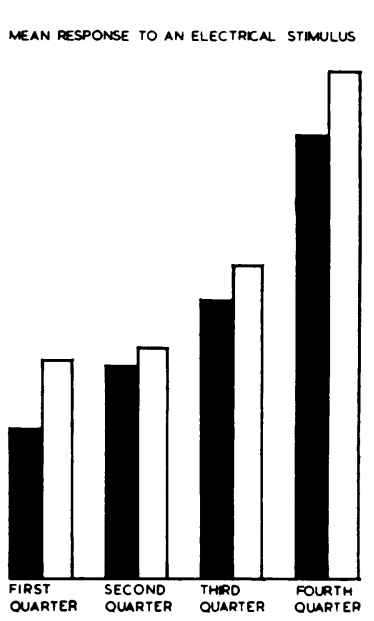

FIG. 2

\section{Correlation Coefficients}

Further evidence concerning the nature of the responses measured in this investigation is provided by the correlations observed between the resting levels of skin resistance and each of the three types of response measured (responses to a deep breath, electrical stimulation, and a warning light). The correlation between skin resistance level and response to a deep breath was $0.585(\mathrm{P}<0.001)$, between skin resistance level and response to shock was $0.579 \quad(\mathrm{P}<0.001)$, whereas the correlation between the skin resistance level and the response to a warning light was only $\mathbf{0 \cdot 0 6 5}$. (This correlation coefficient is not significantly different from zero but is significantly different from both the other two correlation coefficients when $\mathbf{P}<0.05$.) This suggests that the correlation between the resistance level and size of the autonomic response tends to be lower when psychological factors are prominent in determining the response.

If the response to a deep breath is regarded as the best measure of autonomic reactivity to a psychologically neutral stimulus and the response to the warning light as the best measure of autonomic reactivity to a stimulus of emotional significance, the use of the method of partial correlation provides additional information. When the response to light is held constant, the correlation between the resistance level and the response to a deep breath is almost unchanged $(0.582)$; when the response to a deep breath is held constant the correlation between the resistance level and the response to light becomes negative though it is still insignificant at $0 \cdot 016$. Response to a deep breath and response to light would appear to be largely 


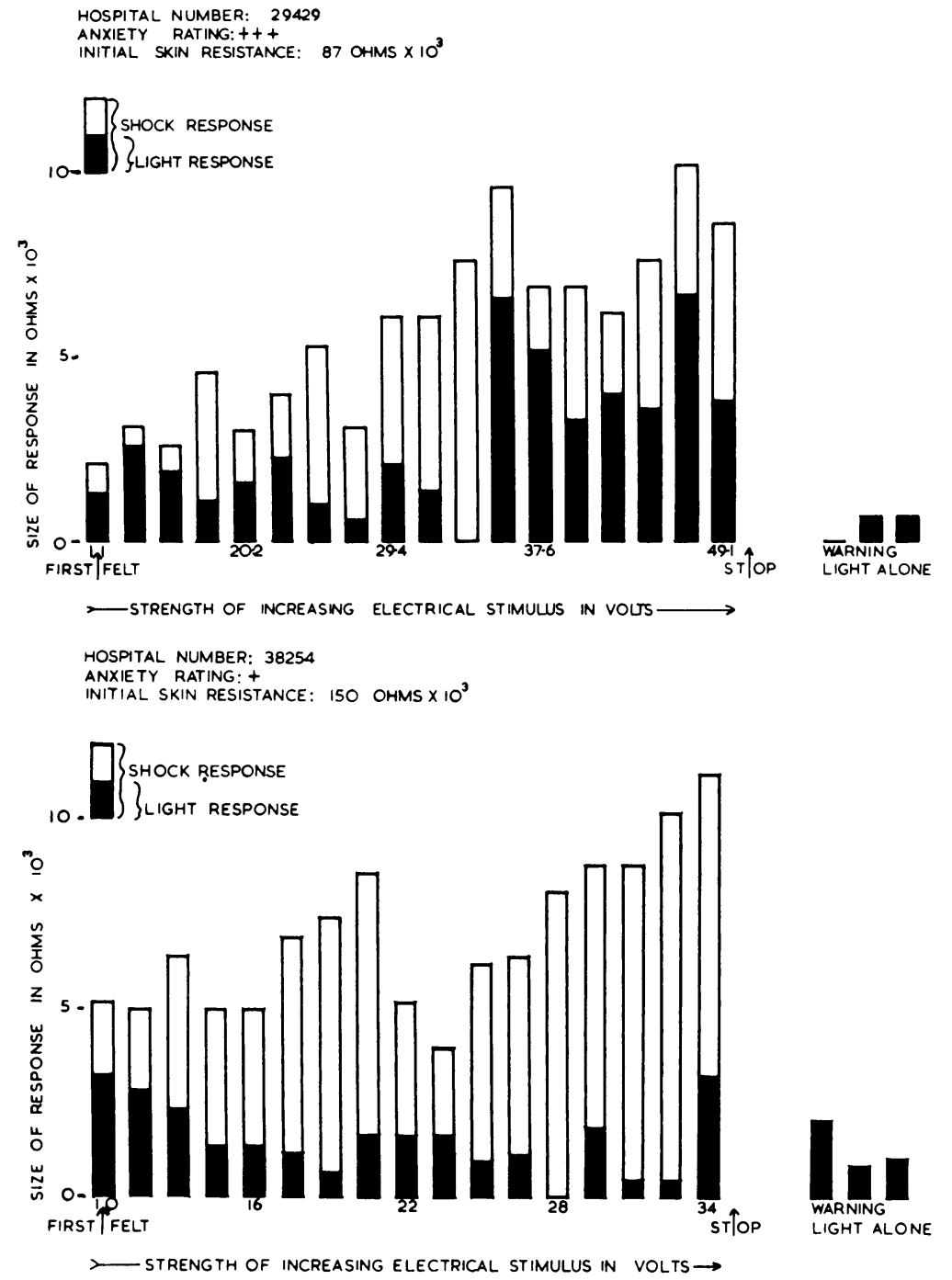

FIGs. $3 a$ and $3 b$

independent. (The actual correlation between these two variables was 0.133 which is not statistically significant.)

It is perhaps worth noting that if the more anxious group is considered alone then the correlation between the resistance level and the response to light, holding the response to a deep breath constant, is 0.384 . This does not quite attain statistical significance but may be regarded as suggestive that the resistance level tends to correlate negatively with a sudomotor response which is determined largely by emotional factors. This would be understandable theoretically and is discussed below.

\section{Discussion}

The results of this study suggest that the determinants of the magnitude of the autonomic responses which we have measured are complex.

It has generally been assumed by previous workers in this field that the size of the psychogalvanic response to a particular stimulus bears a direct relationship to the resting level of skin resistance from which the response starts, and indeed it has been the practice of many investigators to express the response as a percentage of the resting skin resistance level (Haggard, 1949; Malmo and Shagass, 1950 ; Bassett and Ashby, 1954). The 
present results show that this relationship can only safely be assumed with stimuli in which the psychological component is small and constant. Thus in the present investigation there are marked correlations between both the resting level and the responses to a shock (0.579) and between the resting level and the responses to a deep breath $(0.585)$ while the correlation between the resting level and the responses to light is negligible $(0 \cdot 065)$. It would appear therefore that the relationship between the resting skin resistance level (resting sweat rate) and the psychogalvanic response (sudomotor response) depends largely on the nature of the stimulus eliciting the response. In the case of a stimulus which has a minimal emotional significance, such as the deep breath, it seems likely that the size of the response does bear a direct relationship to the resting level of skin resistance. In the case of a stimulus which produces a psychogalvanic response only by virtue of its significance to the subject and not as a result of its own sensory attributes of modality and intensity the situation is more complex. It is reasonable to expect that more anxious people will produce larger responses than less anxious people to emotionally disturbing stimuli, such as the warning light. Now, if heat elimination requirements are kept constant anxious people have lower skin resistance levels than nonanxious people. As a consequence where anxiety is a variable there would be a tendency for the larger responses to be associated with the lower resistance levels. Such a tendency toward a negative correlation might be largely offset by a presumably independent tendency towards a positive correlation. This latter relationship may perhaps be understood most simply if a given stimulus is regarded as a signal tending to cause the organism to reach a certain level of activity rather than as one which causes a certain increase in activity. Thus for a given stimulus low skin resistance levels would be associated with small resistance changes. It is presumably this latter relationship which predominates in the case of the responses to the deep breath and to shock. We suggest therefore that the size and sign of the correlation between psychogalvanic responses and the resting skin resistance depend upon the comparative contribution of emotional or "psychological" mechanisms and more immediate physiological mechanisms.

It is to be expected that emotional factors would be present in the autonomic response to the electrical stimulus. Although this stimulus produces a local reflex response which may even show a local sign (Elithorn and others, 1951) pain is of course necessarily emotionally disturbing. We have evidence also that the electrical stimulus is more disturbing to our more anxious group of patients than to the other group. Thus the ratio of the mean shock response to the mean deep breath response distinguishes clearly between our two groups of patients.

It would seem fair to claim that these observations could be developed to provide a method of estimating the comparative contributions of different levels of autonomic organization in determining the magnitude of a particular autonomic response. In distinguishing between "emotional" and more "local" reflex control of autonomic responses we are, of course invoking a hierarchical organization within the autonomic system and assuming that the " emotional" control of sudomotor responses involves mechanisms which also control many other aspects of behaviour.

There remain to be considered variations within a single test session of the autonomic significance of a physically constant stimulus. We have shown that the ratio of the mean response to light in the fourth quarter to the mean response to light in the first quarter is significantly greater in our more anxious than in our less anxious group. Light stimuli in the fourth quarter differ from light stimuli in the first quarter in two essential ways : position in serial order and the intensity of the electrical stimuli which they precede. By virtue of the factor of position in serial order the higher ratio in the more anxious patients could be accounted for either in terms of poorer habituation or in terms of stronger conditioning. By virtue of the factor of intensity of electrical stimulation the difference between the two groups could be accounted for in terms of accumulating anxiety to an increasingly stressful situation.

We have little evidence to offer as to the importance of conditioning in the Pavlovian sense. We found that responses to the warning light quickly, and often immediately, disappeared when the patient was told there would be no further shocks. This observation is similar to that reported by Mowrer (1938). The responses to a warning light can more fully be described as conditional upon insight and past experience than as simply conditioned by the shock. This does not mean however that conditioning in the Pavlovian sense does not occur. It is possible, for example, that the occurrence of conditioning is merely restricted by other factors such as instructions.

Habituation of the response to light is likely to be important in so far as the light signal is able to initiate an autonomic response before any conditioning has taken place. In ordinary speech we would say that the patient is "anxious" at the 
beginning of the investigation before receiving any painful stimuli, and that during the early part of the session this anxiety somewhat decreases (or habituates) and that response to a warning light diminishes correspondingly.

Conditioning and habituation are mechanisms which are familiar to learning theory and are essentially related to the time relations of stimuli and responses. Apart from these mechanisms it is necessary to consider an " anxious" response to a stressful situation. Such a response may be initiated by the instructions of the examiner before the patient has had any experience of the test situation. It may also be aroused by a change in the significance of the stimulus which elicits the autonomic response. Thus it seems probable that the increase in the size of the response to light as the investigation proceeds results at least as much from the shifting significance of this stimulus as from conditioning in the strict sense. This interpretation is supported by the disappearance of the responses to light when the patient is told that there will be no further shocks. Although there is ample evidence for such a mechanism its nature is obscure and would seem to be more appropriately discussed in relation to such concepts as perception and insight rather than in relation to conditioning and learning as ordinarily understood. It may, for example, be regarded as an amplification of classical conditioning mechanisms just as "imprinting" (Prägung of Lorenz, 1935) may be considered to be a refinement and limitation of an innate response tendency. Indeed Tinbergen (1951) regards imprinting as a type of conditioning.

At all events it may be said that increased conditioning, reduced habituation, and greater reaction to emotional stress are to be expected in the more anxious patients. These three tendencies would all affect the ratio (fourth quarter light response/first quarter light response) under consideration in the same direction, namely in the direction of an increase in the ratio. This ratio is significantly higher in our more anxious than in our less anxious group, and since, as discussed above, there is reason to suppose that this ratio is determined by three mechanisms all acting in the same direction, it is to be expected that it would be a fairly sensitive indicator of anxiety.

Finally it should be mentioned that a similar effect was found in the case of responses to the electrical stimulus. The ratio fourth quarter shock response/first quarter shock response is greater in the more anxious group than in the less anxious group but the difference is not statistically significant. It is relevant also that in both groups the fourth quarter/first quarter ratio is much greater in the case of responses to shock than in the case of responses to light. It seems likely that changes in the " psychological " component of the response to shock are largely masked by the lower level reflex whose magnitude may be expected to depend upon the intensity of the stimulus.

\section{Summary}

The sudomotor responses (psychogalvanic reflexes) of a group of 36 psychiatric patients have been studied. The stimuli used were both physiological and psychological. The pain tolerance of all patients was measured.

Before the investigation each patient was assessed psychiatrically and rated for the level of clinical anxiety present. On this basis the 36 patients were divided into two equal groups, "less anxious" and " more anxious".

The pain tolerance and the sudomotor responses to three types of stimuli-a deep breath, an electric shock, and a warning light-were not significantly하 different for the two groups. However, when the्p psychological responsiveness was considered in? relation to the physiological responsiveness it wase $\vec{z}$ found that the former was relatively much greateb 일 in the more anxious group. This effect was marke and significant beyond the $0 \cdot 1 \%$ level of confidence $\overrightarrow{0} \cdot \overrightarrow{0}$

Some psychological determinants of autonomic or responses are discussed, and it is concluded that under equal conditions of stress the more anxious group were distinguished by a tendency to form anticipatory sets which were accompanied by a relatively greater degree of autonomic disturbance.

We would like to express our gratitude to Dr. E. A. Carmichael, Professor J. Elkes, Dr. Eliot Slater, and Professor O. L. Zangwill, whose help and encouragement made this work possible. To Dr. P. Armitage we are indebted for statistical advice.

\section{REFERENCES}

Bassett, M., and Ashby, W. R. (1954). J. ment. Sci., 100, 632. Elithorn, A., Piercy, M. F., and Crosskey, M. A. (1951). Journal of Neurology, Neurosurgery and Psychiatry, 14, 209.

$\longrightarrow,-$ (1955). Ibid., 18, 34.

Haggard, E. A. (1949). J. exp. Psychol., 39, 378.

Lorenz, K. (1935). J. Orn., Berl., 83, 137-213, 289-413. Malmo, R. B., and Shagass, C. (1950). Arch. Neurol. Psychiat.,

Mowrer, O. H. (1938). Psychol. Rev., 45, 62.
Tinbergen, N. (1951). The Study of Instinct. Clarendon Press, Oxford.

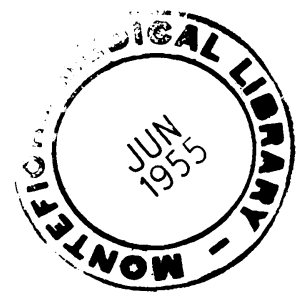

